\title{
SOME REMARKS ON DIFFUSION MODEL OF POSITRON REEMISSION AND/OR POSITRON SURFACE TRAPPING
}

\author{
M. Degbowska and W. ŚwiątKowski \\ Institute of Experimental Physics, Wrocław University \\ Pl. M. Borna 9, 50-204 Wrocław, Poland
}

The problem of a role of boundary conditions in a diffusion model of positron reemission is discussed. It was shown that a sink rate cannot change from zero up to infinity but has an upper limit equal to a half of a mean positron velocity. Some additional relations connected with this result are presented as well.

PACS numbers: $68.35 . \mathrm{Wm}$

\section{Introduction}

In the problem of positron reemission (and/or positron surface trapping) treated as being controlled by positron diffusion the following simple positron fate equation for the interior of a sample (say a reemitter) is often used:

$\frac{\partial c(x, y, z, t)}{\partial t}=S(x, y, z, t)+D \nabla^{2} c(x, y, z, t)-\lambda c(x, y, z, t)-\kappa c(x, y, z, t)$.

The first term in the right part of Eq. (1), $S(x, y, z, t)$, describes the implantation of positrons (implantation profile), the second one - the diffusion determined changes of positron concentration $(c(x, y, z, t))$ and two last terms - the annihilation and trapping processes respectively. $D$ is a positron diffusion constant, $\lambda-$ a positron annihilation rate and $\kappa-$ a positron trapping rate.

As a rule the situation is considered as in Fig. 1. Positrons injected into the sample-reemitter are slowing down in it and, after the thermalization, move diffusively. Some of them end their diffusive movement annihilating in the sample, as free or previously trapped, which is shown as a case $a$. Others reach a sample boundary being then reemitted (case $b$ ) or surfacely trapped. Some of positrons reaching the boundary can be reflected and thus they may continue the diffusive movement (case $c$ ) etc. 


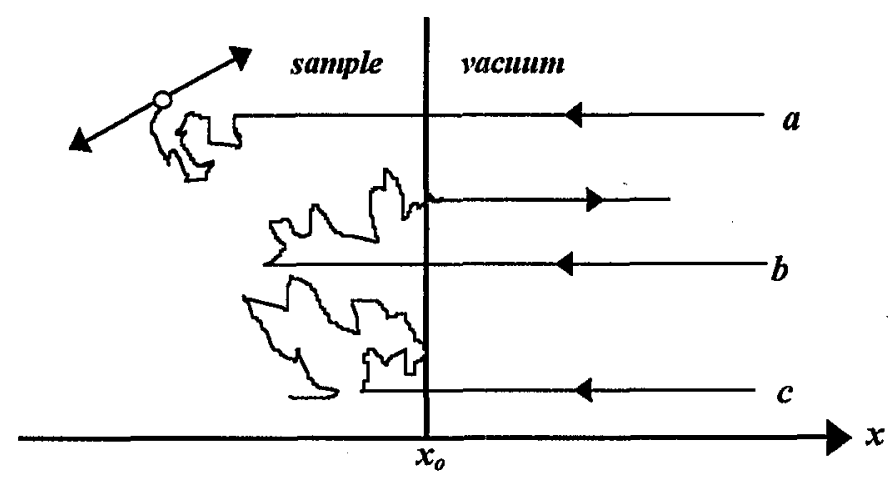

Fig. 1. Simplified presentation of the positron-reemission problem; positrons are injected into the sample through a flat boundary perpendicular to a certain $x$-axis.

Additionally, the assumption is taken that the geometry and positron source characteristics do not depend on time, and thus Eq. (1) can be replaced by the following one-dimensional equation for an equilibrium positron concentration $c(x)$ :

$$
D \frac{\mathrm{d}^{2} c(x)}{\mathrm{d} x^{2}}-(\lambda+\kappa) c(x)+S(x)=0 .
$$

Equation (2) is often used in the form

$$
\frac{\mathrm{d}^{2} c(x)}{\mathrm{d} x^{2}}-\frac{1}{L^{2}} c(x)+\frac{S(x)}{D}=0,
$$

where

$$
L=\sqrt{\frac{D}{\lambda+\kappa}}
$$

is the effective positron diffusion length.

When the solution of Eq. (3) is known then the intensity of the reemission current $\left(J_{\text {reem }}\right)$ can be found using the Fick law, namely

$$
J_{\text {reem }}=-\left.D \frac{\mathrm{d} c(x)}{\mathrm{d} x}\right|_{x=x_{0}},
$$

which gives for a reemission probability the expression

$$
\varepsilon_{\text {reem }}=\frac{J_{\text {reem }}}{\int_{-\infty}^{x_{0}} S(x) \mathrm{d} x} .
$$

When solving Eq. (3) a boundary condition of the form

$$
-\left.D \frac{\mathrm{d} c(x)}{\mathrm{d} x}\right|_{x=x_{0}}=\alpha c\left(x_{0}\right) \text {, }
$$

is commonly used. In Eq. (7) $\alpha$ having a dimension of velocity is called a sink rate and depends on boundary characteristics. It is also assumed that $\alpha$ can change from zero for a perfectly reflecting boundary to infinity for a boundary being perfectly transparent (and/or absorbing) for positrons (see e.g. Britton [1] and references therein). It should be mentioned that for $\alpha=+\infty$ Eq. (7) needs the equilibrium positron concentration $c\left(x_{0}\right)$ equal to zero. 
In this paper we would like to pay one's attention that the assumption of $\alpha$ being infinite is unphysical one.

\section{Sink rate for completely transparent boundary}

Let us start with considering the sample for which $c(x)=$ const. If we take into account the flat surface $x=$ const then diffusing positrons are going through it with equal current densities in both directions. As it is shown in the neutron physics analysis (see. e.g. Beckurts and Wirtz [2]) these densities, say $J_{+}$for $v_{x}>0$, . i.e. for positrons going through the surface in $x$-axis direction and $J_{-}$(for $v_{x}<0$ ) are equal to

$$
J_{+}=J_{-}=\alpha_{0} c(x)
$$

where

$$
\alpha_{0}=\frac{1}{2} \bar{v}_{x^{+}}=\frac{1}{4} \bar{v}
$$

$\bar{v}_{x}+$ denotes the mean value of $x$-components of positron velocities, for positrons having $v_{x}>0$ and $\bar{v}$ is a mean value of velocities of diffusing positrons.

Let us now suppose that for some $x$ value $\mathrm{d} c(x) / \mathrm{d} x \neq 0$, say it is negative. Then we should expect that $J_{+}$increases and $J_{-}$decreases as compared to those given by Eq. (8), namely we should have

and

$$
J_{+}=\alpha_{0} c(x)-\frac{D}{2} \frac{\mathrm{d} c(x)}{\mathrm{d} x}
$$

$$
J_{-}=\alpha_{0} c(x)+\frac{D}{2} \frac{\mathrm{d} c(x)}{\mathrm{d} x}
$$

where $D$ is the diffusion constant. Because the resulting diffusion current density $J$ is the difference of $J_{+}$and $J_{-}$one can easy prove that Eqs. (10) and (11) are the components to the Fick law, namely we have

$$
J(x)=J_{+}-J_{-}=-D \frac{\mathrm{d} c(x)}{\mathrm{d} x} .
$$

Let us now assume that we have a flat boundary, completely transparent for positrons, at $x=x_{0}$ (say as in Fig. 1), the sample being for $x \leq x_{0}$ and the vacuum for $x>x_{0}$. Thus at $x=x_{0}$ we should have no positron current in the direction opposite to the $x$-axis direction $\left(J_{-}=0\right)$ and thus resulting current should be equal to $J_{+}$given by Eq. (10) with $x=x_{0}$. On the other hand, this current should correspond to the Fick law, and thus we should have

$$
J\left(x_{0}\right)=\alpha_{0} c\left(x_{0}\right)-\left.\frac{D}{2} \frac{\mathrm{d} c(x)}{\mathrm{d} x}\right|_{x=x_{0}}=-\left.D \frac{\mathrm{d} c(x)}{\mathrm{d} x}\right|_{x=x_{0}} .
$$

From (13), using (9), we can easily obtain that

$$
J\left(x_{0}\right)=2 \alpha_{0} c\left(x_{0}\right)=\frac{1}{2} \bar{v} c\left(x_{0}\right) \text {. }
$$

It means that the sink rate has an upper limit $\alpha_{\max }$ equal to a half of the mean value of the velocities of the diffusing positrons i.e.

$$
\alpha_{\max }=\frac{1}{2} \bar{v} \text {. }
$$




\section{Sink rate for reflecting boundary}

Let us now consider the boundary (at $x=x_{0}$ ) partially reflecting positrons which reach it, say with a probability equal to $p_{\mathrm{r}}=1-p_{0}$; thus $p_{0}$ is the probability that a positron impinging the boundary does not return to the sample interior (it is removed out of the sample interior). Thus at $x=x_{0}, J_{+}$is determined by Eq. (10) whereas for $J_{-}$we have

$$
J_{-}\left(x_{0}\right)=\left(1-p_{0}\right) J_{+}\left(x_{0}\right)=\left(1-p_{0}\right)\left[\frac{\bar{v}}{4} c\left(x_{0}\right)-\left.\frac{D}{2} \frac{\mathrm{d} c(x)}{\mathrm{d} x}\right|_{x=x_{0}}\right] .
$$

Taking into account the Fick law (12) one can easily obtain that

$$
-\left.D \frac{\mathrm{d} c(x)}{\mathrm{d} x}\right|_{x=x_{0}}=\left[\frac{\bar{v}}{4}\left(\frac{2 p_{0}}{2-p_{0}}\right)\right] c\left(x_{0}\right) \text {. }
$$

It means that the positron sink rate $\alpha$ is connected with the probability $p_{0}$ by the formula

$$
\alpha=\frac{\bar{v}}{4}\left(\frac{2 p_{0}}{2-p_{0}}\right)
$$

which gives $\alpha=0$ for $p_{0}=0$ and $\alpha=\bar{v} / 2$ for $p_{0}=1$ (in agreement with formula (15)).

If there are some possibilities of sinking for positrons reaching the boundary, say a reemission, surface trapping, and positronium formation, then $p_{0}$ should be treated as the sum

$$
p_{0}=p_{\text {reem }}+p_{\text {trapp }}+p_{\text {Ps }}
$$

of the respective partial probabilities. In the literature the sink rate $\alpha$ is often presented as the sum of some $\alpha_{i}$ ascribed to different sinking processes, say in the form (see e.g. [3, 4])

$$
\alpha=\alpha_{\text {reem }}+\alpha_{\text {trapp }}+\alpha_{\mathrm{Ps}} .
$$

It should be however mentioned that a change of the possibility of one of the sink processes changes not only a "sink rate" $\alpha_{i}$ formally ascribed to this process but affects the other of them as well. It is not so in the case of probabilities $p_{i}$ in Eq. (19). Thus $p_{0}$ and its components are more suitable for interpretation than the respective $\alpha$ values.

\section{Probability of positron reemission}

By solving the positron fate Eq. (3) with $S(x)$ (implantation profile) being different from zero only near the distance $z$ from the boundary and using the boundary condition (7) with the assumption that the only sink process is the reemission, one can easy obtain the known formula (see e.g. [1]) for the probability of the reemission of a positron being implanted at the distance $z$ from the boundary, namely

$$
\varepsilon(z)=\frac{\alpha}{\alpha+D / L} \mathrm{e}^{-z / L}
$$


Taking in (21) $\alpha$ equal to infinity we obtain

$$
\varepsilon_{\max }(z)=\mathrm{e}^{-z / L},
$$

which is interpreted as the probability of the positron reemission for the case of the perfectly transparent (not reflecting) reemitter boundary. By taking into account that $\alpha$ has the upper limit, given by formula (15), we should replace (22) with formula

$$
\varepsilon_{\max }(z)=\frac{\alpha_{\max }}{\alpha_{\max }+D / L} \mathrm{e}^{-z / L}=\frac{\bar{v}}{\bar{v}+2 D / L} \mathrm{e}^{-z / L} .
$$

The differences between values $\varepsilon_{\max }(z)$ resulting from (22) and (23) are not very significant ones. For example if we take $D=10^{-4} \mathrm{~m}^{2} \mathrm{~s}^{-1}, L=10^{-7} \mathrm{~m}$ and $\bar{v}=10^{5} \mathrm{~m} \mathrm{~s}^{-1}$ (approximately the mean value of the thermalized positron velocities at room temperature) the preexponential term in (23) appears to be close to 1 , namely

$$
\frac{\bar{v}}{\bar{v}+2 D / L} \approx 0.98 \text {. }
$$

This term can be as well treated as the upper limit of a positron reemission probability related to a positron implanted just near the reemitter boundary. Why is it thus different from 1 ? For a positron implanted near the perfectly transparent reemitter boundary there are equal to $1 / 2$ both the probability of impinging the boundary and escaping from the reemitter, and the probability of starting to move with such velocity direction as if it was reflected from the boundary. In the last case the positron will be some time in a diffusive movement which can be stopped, say with a probability $1-\gamma$, by its annihilation in the reemitter interior or, with a probability equal to $\gamma$, by its reaching the boundary and escaping from the reemitter. Thus we have a relation

$$
\frac{\bar{v}}{\bar{v}+2 D / L}=\frac{1}{2}+\frac{1}{2} \gamma \text {. }
$$

Solving (25) with respect to $\gamma$ we obtain the formula

$$
\gamma=\frac{\alpha_{\max }-D / L}{\alpha_{\max }+D / L}=\frac{1-2 D / L \bar{v}}{1+2 D / L \bar{v}},
$$

which gives us the probability that the positron reflected from the boundary reaches it once more. For the parameter values used in $(24)$ we obtain $\gamma \approx 0.96$. It means that for typical sample conditions the positron reflected from the boundary has a relatively high probability to return to it once more.

Let us now return to the situation when the positron impinging the boundary can escape (sink) with the probability $p_{0}$. The formula (23) gives us the probability that positron implanted at the distance $z$ from the boundary impinges it at least once. According to this the common probability of the reemission, for such positron, can be written as the sum

$$
\begin{aligned}
\varepsilon(z) & =p_{0} \varepsilon_{\max }(z)+p_{0} \varepsilon_{\max }(z)\left[\left(1-p_{0}\right) \gamma\right]+p_{0} \varepsilon_{\max }(z)\left[\left(1-p_{0}\right) \gamma\right]^{2}+\ldots \\
& =p_{0} \varepsilon_{\max }(z)\left\{1+\left[\left(1-p_{0}\right) \gamma\right]+\left[\left(1-p_{0}\right) \gamma\right]^{2}+\ldots\right\} \\
& =\varepsilon_{\max }(z) \frac{p_{0}}{1-\left(1-p_{0}\right) \gamma}
\end{aligned}
$$


This formula, being equivalent to Eq. (21) with $\alpha$ given by (18) has the form more suitable to physical interpretation than Eq. (21). Here the probability of reaching the boundary by the positron implanted at the distance $z$ from it $\left(\varepsilon_{\max }(z)\right)$ and the probability that the positron reflected from the boundary returns to it $(\gamma)$ do not depend on the boundary conditions. These last ones are represented in formula (27) by the parameter $p_{0}$.

\section{Final remarks}

The fact, pointed out in the paper, that a sink rate has an upper limit, changes some existing views related to the diffusion model of a positron reemission. We limit us here to one example. Britton [1] has written that for a perfectly transparent boundary, that is for $\alpha=\infty$, the reemission current is determined by the gradient of the positron concentration only. In fact, as it is seen from (13) this gradient can give only a half of the reemission intensity needed by the Fick law.

\section{Acknowledgment}

This work was supported by University of Wrocław, grant No. 2016/IFD/98.

\section{References}

[1] D.T. Britton, in: Positron Beams for Solids and Surfaces, AIP Conf. Proc. 218, Eds. P.J. Schultz, G.R. Massoumi, P.J. Simpson, American Institute of Physics, New York 1990, p. 207.

[2] K.H. Beckurts, K. Wirtz, Neutron Physics, Springer-Verlag, Berlin 1964.

[3] J. Mäkinen, P.A. Huttanen, D.T. Briton, E. Soininen, A. Vehanen, in Ref. [1], p. 199.

[4] Y. Kong, R.M. Nieminen, P.A. Huttunen, A. Vehanen, J. Mäkinen, in Ref. [1], p. 91. 